"This document is the Accepted Manuscript version of a Published Work that appeared in final form in The Journal of Physical Chemistry A, copyright (C) American Chemical Society after peer review and technical editing by the publisher. To access the final edited and published work see https://pubs-acs-org.uml.idm.oclc.org/doi/10.1021/jp507018a”.

\title{
Microwave Spectroscopic Investigation and Structural Determination of the Ar-Difluoropyridine van der Waals Complexes
}

\author{
Ming Sun ${ }^{1}$, Mahdi Kamaee ${ }^{2}$ and Jennifer van Wijngaarden ${ }^{2 *}$ \\ ${ }^{1}$ School of Electronic and Optical Engineering, Nanjing University of Science and \\ Technology, Xiao Lingwei Road 200, Nanjing, Jiangsu, 210094 China \\ ${ }^{2}$ Department of Chemistry, University of Manitoba, Winnipeg Manitoba, R3T 2N2 \\ Canada \\ *Corresponding author \\ Email: vanwijng@cc.umanitoba.ca
}

Phone: (204)474-8379

Fax: (204)474-7608 


\begin{abstract}
The ground state rotational spectra of the van der Waals dimers of 2,3-, 2,4-, 2,5-, 2,6-, and 3,5-difluoropyridine with argon were investigated in the range of 4 to $26 \mathrm{GHz}$ using both Fourier transform microwave (FTMW) and chirped pulse Fourier transform microwave (cp-FTMW) spectroscopy. The spectroscopic parameters derived from fitting the observed transitions were used to estimate the van der Waals coordinates of the five weakly bound complexes and to quantitatively describe their internal dynamics using a pseudodiatomic model. The experimental structures are in fair agreement with MP2 calculations $(6-311++\mathrm{G}(2 \mathrm{~d}, 2 \mathrm{p}))$ and have the centers of mass of the two moieties separated by 3.486 to $3.545 \AA$ with the Ar atom lying $2-10^{\circ}$ from the $\mathrm{c}^{\prime}$ axis of the ring monomer.
\end{abstract}

KEYWORDS: Fourier transform microwave spectroscopy; van der Waals complex; aromatic ring; difluoropyridine; pseudodiatomic model. 


\section{INTRODUCTION}

A number of van der Waals complexes formed by aromatic rings and rare gas atoms have been the subject of investigation via rotational spectroscopy. ${ }^{1,2,3,4,5,6}$ Complexes of pyridine with $\mathrm{He}, \mathrm{Ne}, \mathrm{Ar}$ and $\mathrm{Kr}$ have been studied, for example, and serve as useful prototypes as the pure rotational transitions are strong due to the large dipole moment of the pyridine subunit. ${ }^{1,2,7,8,9,10}$ The structure and internal dynamics of weakly bound dimers may be explored through interpretation of the experimentally-derived spectroscopic constants using a pseudodiatomic model in which the monomer is treated as a rigid entity while the complex itself undergoes low frequency vibrations. For the pyridine-containing dimers, the ${ }^{14} \mathrm{~N}$ nuclear quadrupole hyperfine structure also provides a local probe of the electronic environment near the nitrogen atom which affords an alternative method for analyzing vibrationally-averaged structures of weakly bound systems. ${ }^{10}$

We recently reported the results of our investigation of the van der Waals dimers of Ar with 2- and 3-fluoropyridine using both chirped pulse Fourier transform microwave (cp-FTMW) and Balle-Flygare-type FTMW spectrometers in conjunction with ab initio calculations. ${ }^{10}$ It was found that with the Ar atom sitting above the aromatic ring plane, the center of mass separation of the two moieties in the dimers is between 3.5 and $3.6 \AA$ and the $\mathrm{Ar}$ atom lies $5^{\circ}$ to $11^{\circ}$ off the monomer c'-axis toward the nitrogen atom in the pyridine backbone. Compared to the unsubstituted pyridine-Ar dimer, single fluorine substitution on the aromatic ring tends to lower the pseudodiatomic stretching mode and this effect is more significant for fluorine substitution at the meta position (3-fluoropyridine) rather than at the ortho position (2-fluoropyridine). This was attributed 
to subtle differences in the electronic structure of the $\pi$-bonding system of the two rings caused by the hyperconjugation effect which stabilizes the 2-fluoropyridine monomer itself. ${ }^{11}$ The results suggest that the van der Waals dimer with fluorination at the ortho carbon is more strongly bound than the analog substituted at the meta position.

In this paper, we systematically extend our study to a series of difluoropyridine-Ar complexes whose transitions were first identified in broadband rotational spectra recorded in the course of our investigation of the difluoropyridine monomers. ${ }^{12} \mathrm{We}$ report here the first rotational spectra of five van der Waals Ar dimers with 2,3-, 2,4-, 2,5-, 2,6- and 3,5-difluoropyridines along with ab initio estimates of their geometries at the MP2 (6-311G++(2d,2p)) level of theory. Double fluorination of the aromatic pyridine subunit provides an opportunity to explore a broader variation of structural effects when compared with Ar complexes of the unsubstituted ${ }^{1,2}$ and mono-substituted ${ }^{10}$ pyridine analogs. The results of our spectroscopic investigation, including detailed analyses of inertial moments and the observed ${ }^{14} \mathrm{~N}$ hyperfine structure, are interpreted in terms of a pseudodiatomic model of the van der Waals system which allows systematic comparison of the structures and dynamics with related weakly bound complexes.

\section{EXPERIMENT}

The pure rotational spectra of the Ar complexes with 2,3-, 2,4-, 2,5-, 2,6- and 3,5-difluoropyridine were first observed using the broadband chirped pulse Fourier transform microwave (cp-FTMW) instrument in the van Wijngaarden group at University of Manitoba which has been previously described. ${ }^{13}$ Survey spectra were measured in 2 GHz windows between 8-16 GHz and the observed transitions had linewidths of $\sim 200 \mathrm{kHz}$ 
FWHM when individual FIDs were acquired for $20 \mu$ s. Liquid samples of each compound (2,6-difluoropydrine: Sigma Aldrich, 99\%; all others from Synthonix, 95\% of better) were purchased and prepared as a gas mixture of about $0.05 \%$ difluoropyridine in argon and delivered to the pulsed nozzle with a backing pressure of $20 \mathrm{psi}$ or $138 \mathrm{kPa}$. For each single gas pulse, multiple FIDs were recorded to improve the efficiency of signal acquisition and reduce sample consumption. In general, 50000 shots with 15 FIDs per shot were averaged for each $2 \mathrm{GHz}$ window. These initial survey spectra guided our experiments using the more sensitive Balle-Flygare FTMW spectrometer ${ }^{14}$ which was used to record individual hyperfine components with higher resolution ( $\sim 7 \mathrm{kHz}$ FWHM) and to seek lower intensity transitions falling in the range 4-26 GHz. Gas mixtures for the FTMW measurements were more dilute $(0.01 \%)$ and maintained at a higher backing pressure ( $80 \mathrm{psi}$ or $550 \mathrm{kPa}$ ) to optimize the signal.

\section{THEORETICAL CALCULATIONS}

The equilibrium geometries of the difluoropyridine-Ar dimers were estimated at the MP2 level $(6-311++\mathrm{g}(2 \mathrm{~d}, 2 \mathrm{p}))$ using the Gaussian09W software package. ${ }^{15}$ The five monomer geometries were taken from our earlier ab initio study of the difluoropyridines ${ }^{12}$ and were assumed to be unaffected by complexation with Ar. A series of counterpoise-corrected single point energy calculations was done for each dimer to sample the potential energy surface for geometries with the Ar atom above the monomer plane which was consistent with the observed pattern of transitions in the cp-FTMW spectrum. Three parameters were used to describe the orientation of $\mathrm{Ar}$ above the ring: $\mathrm{R}$ (the distance between the Ar atom and the center of mass (CM) of the monomer ring), $\theta$ (the 
Ar-CM-N angle) and $\phi$ (the angle between the plane of the ring and the plane containing Ar-CM-N). A large grid scan was initially done using increments of $0.1 \AA$ in $\mathrm{R}$ and $5^{\circ}$ in the two angles sampling van der Waals separations of 3.4-3.7 $\AA$ and angles of $70-110^{\circ}$. Based on these first points, finer steps $\left(0.01 \AA\right.$ in $\mathrm{R}$ and $1^{\circ}$ in the two angles) were used to find the minimum energy geometry. The resulting geometries are schematically shown in Figure 1 looking down on the ab-planes of the dimers. These correspond to values of $R, \theta, \phi$ of $\left(3.56 \AA, 82^{\circ}, 84^{\circ}\right),\left(3.56 \AA, 83^{\circ}, 87^{\circ}\right),\left(3.52 \AA, 86^{\circ}, 89^{\circ}\right),\left(3.51 \AA, 92^{\circ}, 90^{\circ}\right)$, and $(3.57 \AA$, $81^{\circ}, 90^{\circ}$ ) for the 2,3-, 2,4-, 2,5-, 2,6- and 3,5-difluoropyridine complexes with Ar, respectively. Previous calculations for Ar complexes with pyridine, 2-fluoropyridine and 3-fluoropyridine yielded values of $\left(3.55 \AA, 86^{\circ}, 90^{\circ}\right),\left(3.54 \AA, 88^{\circ}, 85^{\circ}\right)$, and $\left(3.58 \AA, 82^{\circ}\right.$, $\left.86^{\circ}\right)$, respectively.

\section{RESULTS AND ANALYSIS}

During our investigation of the five difluoropyridine monomers and their minor ${ }^{13} \mathrm{C}$ and ${ }^{15} \mathrm{~N}$ isotopologues, ${ }^{12}$ additional weak transitions were observed in the broadband cp-FTMW spectrum of each species, which disappeared once the carrier gas was changed from argon to neon as shown in Figure 2. These transitions were subsequently assigned to the van der Waals dimers of the difluoropyridine rings with Ar. Using the cp-FTMW spectra as a guide for each, we used our Balle-Flygare FTMW instrument to measure the

${ }^{14} \mathrm{~N}$ hyperfine splitting of these and other transitions. Spectra arising from minor isotopologues of the van der Waals dimers were not observed in the cp-FTMW spectra and were not sought in the subsequent higher resolution experiments as the observed intensities of the parent species were fairly unstable. 


\section{i) 2,3-difluoropyridine-Ar}

Based on ab initio calculations at the MP2 level, 2,3-difluoropyridine-Ar has strong dipole components along the b- and c- principal inertial axes $\left(\mu_{\mathrm{b}}=3.4 \mathrm{D}\right.$ and $\mu_{\mathrm{c}}=$ $2.4 \mathrm{D})$ with a negligible component along the van der Waals axis $\left(\mu_{\mathrm{a}}=0.02 \mathrm{D}\right)$. As a result, both $b$-type and $c$-type transitions were observed for the complex and a sample FTMW spectrum for 2,3-difluoropyridine-Ar showing the ${ }^{14} \mathrm{~N}$ nuclear quadrupole hyperfine structure is given in Figure 3. In total, 352 hyperfine components from 125 different rotational transitions (74 $b$-type and $51 c$-type) of the parent complex were measured between 5 and $22 \mathrm{GHz}$. The observed transitions in this region span quantum numbers $\mathrm{J}^{\prime}=$

2 through $\mathrm{J}^{\prime}=10$. The ${ }^{14} \mathrm{~N}$ hyperfine constants of 2,3-difluoropyridine-Ar were calculated in G09 at the minimum energy geometry using the keyword Pickett and the off-diagonal nuclear quadrupole coupling constants were found to be significant although the observed transitions were not particularly sensitive to these parameters. The spectra were fit in Pickett's SPFIT program ${ }^{16}$ using Watson's A-reduced Hamiltonian ( $I^{r}$ representation) with the off-diagonal components of the nuclear quadrupole coupling tensor fixed at the $a b$ initio values. The inclusion of the off-diagonal terms did not influence the quality of the fit or the values of the other parameters as the transitions measured were not sensitive to these terms. The spectroscopic constants are reported in Table 1 including standard errors from Kisiel's PIFORM program. ${ }^{17}$ The observed transition frequencies are provided as supplemental data.

\section{ii) 2,4-difluoropyridine-Ar}


As in the previous case, 2,4-difluoropyridine-Ar has significant dipole components running perpendicular to the van der Waals a-axis: $\mu_{\mathrm{b}}=2.3 \mathrm{D}$ and $\mu_{\mathrm{c}}=0.5 \mathrm{D}$ and the assignment followed that described above. In total, $76 b$-type and $26 c$-type rotational transitions consisting of 295 hyperfine components were measured for the parent species, spanning quantum numbers $\mathrm{J}^{\prime}=2$ through $\mathrm{J}^{\prime}=9$. The spectroscopic constants are reported in Table 1.

iii) 2,5-difluoropyridine- $\mathrm{Ar}$

For 2,5-difluoroprydine-Ar, the calculated dipole has strong components along the b- and c- principal inertial axes: $\mu_{\mathrm{b}}=1.3 \mathrm{D}$ and $\mu_{\mathrm{c}}=2.3 \mathrm{D}$. The 240 hyperfine lines measured for the parent species arise from $33 b$-type and $50 c$-type rotational transitions, spanning $\mathrm{J}^{\prime}=3$ through $\mathrm{J}^{\prime}=9$. A complete set of spectroscopic constants is shown in Table 1.

\section{iv) 2,6-difluoropyridine-Ar}

Due to additional symmetry, 2,6-difluoropyridine is calculated to have a dipole with a strong component along only the c- principal inertial axis $\left(\mu_{c}=4.5 \mathrm{D}\right)$ and thus, 73 $c$-type rotational transitions yielding 227 hyperfine components were measured. These spanned quantum numbers $\mathrm{J}^{\prime}=2$ through $\mathrm{J}^{\prime}=9$ and the resulting spectroscopic parameters are reported in Table 1.

\section{v) 3,5-difluoropyridine- $A r$}


The symmetry of 3,5-difluoropyridine-Ar is analogous to that of 2,6-difluoropyridine-Ar but its dipole is calculated to be much smaller $\left(\mu_{\mathrm{c}}=0.5 \mathrm{D}\right)$. Nevertheless, 152 hyperfine components arising from $51 c$-type rotational transitions were observed, spanning $\mathrm{J}^{\prime}=3$ through $\mathrm{J}^{\prime}=8$. The lower intensities made it difficult to measure the lower and higher $\mathrm{J}^{\prime}$ transitions as observed for the other difluoropyridine-Ar dimers leading to a smaller data set. The spectroscopic constants are reported in Table 1.

\section{DISCUSSION}

For van der Waals dimers, structural estimates can be obtained using Kisiel's RGDFIT 10,17, 18,19 program or "extreme Kraitchman" analysis, ${ }^{10,20,21}$ even when transitions due to minor isotopologues are not observed. These approaches assume that the geometry of the molecular monomer(s) are unaffected upon complexation such that only the van der Waals coordinates that define the center of mass (CM) separation and relative orientation of the monomers are extracted from the spectroscopic constants to define the geometry. In these methods, the absolute values of three dimer coordinates are fitted to the three rotational constants of the complex via a least squares routine. This yields multiple possible structures that reproduce the same set of rotational constants. Using ab initio structures as a guide, however, one can determine the most likely geometry. We have followed this procedure using Kisiel's RGDFIT program to determine the geometry of each difluoropyridine-Ar dimer. The final results are shown in Table 2 along with the structural parameters of pyridine-Ar and furan-Ar for comparison. ${ }^{1}$ Although only three coordinates can be derived from the spectra, we have reported four parameters (derived from these three) for ease of interpretation across the 
full set of complexes which have different $\mathrm{CM}$ and principal inertial axis orientations. The coordinates reported $(\mathrm{R}, \gamma, \theta, \phi)$ are described pictorially in Figure 4 such that $\mathrm{R}$ is the distance from Ar to the center of mass $(\mathrm{CM})$ of the monomer ring, $\gamma$ is the angle between the $\mathrm{c}^{\prime}$ axis of the monomer and the Ar-CM line, $\theta$ is the Ar-CM-N angle and $\phi$ is the angle between the Ar-CM-N plane and the monomer ring plane (using CM-N-C5 for definition), as reported previously for the 2- and 3-fluoropyridine-Ar complexes. ${ }^{10}$ Based on the results in Table 2, the Ar atom is located 3.486 to $3.545 \AA$ above the aromatic ring with the Ar-CM line lying almost perpendicular to the ring plane $\left(\gamma\right.$ ranging from $2^{\circ}$ to $10^{\circ}$ ). In most cases, the Ar-CM line tilts slightly towards the heteroatom (angle $\theta$, $\angle \mathrm{Ar}-\mathrm{CM}-\mathrm{N}$ or $\angle \mathrm{Ar}-\mathrm{CM}-\mathrm{O}$, ranging from $80^{\circ}$ to $87^{\circ}$ ). The only exception to this is the 2,6-difluoropyridine- $\operatorname{Ar}$ dimer $\left(\theta=94.9^{\circ}\right.$ from RGDFIT analysis) in which the $\mathrm{CM}$ of the monomer ring is shifted closer to the nitrogen due to the location of the two heavy fluorine atoms.

For comparison, the ab initio results for all difluoropyridine-Ar dimers in terms of the same four coordinates are also listed in Table 2. In general, the structural parameters from both the ab initio and inertial analysis methods are consistent with only a few degrees difference in the van der Waals angles and a few hundredths of an Angstrom difference in the van der Waals bond lengths. Such tiny discrepancies may be a result of large amplitude zero-point motions which are not accounted for in the ab initio estimates of the equilibrium geometry. Upon complexation, the three translational degrees of freedom of the Ar atom are transformed into three van der Waals vibrational modes: the two bending modes around the monomer principal axes $a^{\prime}$ and $b^{\prime}$, as well as the pseudodiatomic stretching mode between the $\mathrm{CM}$ of the monomer and the Ar atom. 
Although the RGDFIT program fits three van der Waals coordinates to experimental rotational constants, the contribution of large amplitude motions is not included in such an analysis.

As shown earlier for the fluoropyridine-Ar dimers ${ }^{10}$ and other nitrogen bearing rare-gas complexes, ${ }^{1,18,21,22}$ the dimer geometry can also be evaluated through analysis of the ${ }^{14} \mathrm{~N}$ quadrupole coupling constants. For a rigid aromatic ring weakly bound to a rare gas atom, the electric field gradients in the monomer can be assumed intact upon the complexation. In that case, if the ${ }^{14} \mathrm{~N}$ quadrupole coupling constants in the principal inertial frame are known for both the monomer and the dimer, one can easily deduce the rotation matrix between the monomer $\left(a^{\prime}, b^{\prime}, c^{\prime}\right)$ and the dimer $(a, b, c)$ principal inertial frames. With this matrix, the Ar-CM distance obtained from the RGDFIT analysis and the $\mathrm{r}_{0}$ structure of the monomer, ${ }^{12}$ we derived a second set of van der Waals coordinates to describe the position of the Ar atom in the dimers with the difluoropyridines. These are included in Table 2 for comparsion. Generally, the structures determined via the ${ }^{14} \mathrm{~N}$ hyperfine analysis are in better agreement with the MP2 calculations than those derived via the inertial analysis but this is a consequence of including the $a b$ initio derived off-diagonal quadrupole coupling constants which obviously have a strong influence on the rotation matrix. ${ }^{10}$

In Table 3, we list the experimental and theoretical ${ }^{14} \mathrm{~N}$ quadrupole coupling constants of the complexes under study for direct comparison. Using the rotation matrix from the RGDFIT analysis directly, the quadrupole coupling tensor of the monomer can be rotated into the principal inertial axis system of the dimer to verify the dimer geometry. The quadrupole coupling constants obtained by this method show reasonable agreement 
with both MP2 calculations and spectral fitting results and are listed in Table 3. It might be worth mentioning that for 2,6- and 3,5-difluoropyrine-Ar dimers, the experimental $\chi_{\mathrm{bb}}$ value is virtually the same as the $\chi_{a^{\prime} a^{\prime}}$ of the corresponding monomer due to the additional molecular symmetry of the ring moiety and complex (1.84 MHz for 2,6-difluoropyrine and 1.52 MHz for 3,5-difluoropyrine).

In order to evaluate the pseudodiatomic stretching mode of an aromatic ring-Ar complex, the dimer may be treated as two separate subunits, the monomer ring and $\mathrm{Ar}$ atom. ${ }^{3,10}$ The pseudodiatomic stretching force constant $k_{\mathrm{s}}$ can be calculated from the dimer's centrifugal distortion constant $\Delta_{\mathrm{J}}$ when the Ar-CM line is nearly aligned with the dimer's $a$-axis: $: 3,10,23,24$

$$
k_{s}=\frac{16 \pi^{4} \mu^{2} R^{2}}{h \Delta_{J}}\left[\left(B^{2}+C^{2}\right)^{2}+2\left(B^{4}+C^{4}\right)\right]
$$

where $\mu$ is the reduced mass of the dimer when treated as two pseudodiatomic subunits; $\mathrm{R}$ $\left(\mathrm{R}_{0}\right.$ in Table 4$)$ is the distance from the $\mathrm{CM}$ of the monomer to the Ar atom determined by the inertial analysis; $\mathrm{B}$ and $\mathrm{C}$ are the rotational constants of the dimers. With the force constant $k_{\mathrm{s}}$ and the pseudodiatomic distance $R$, the pseudodiatomic stretching frequency $v_{\mathrm{s}}$ as well as the Lennard-Jones potential well depth $\epsilon$ can also be evaluated ${ }^{3,23}$ as listed in Table 4. In the same table, the analogous potential parameters of pyridine-Ar and furan-Ar are also provided for comparison..$^{1,3}$

Compared to the other difluoropyridine-Ar dimers, the stretching mode in 2,6-difluoropyrine-Ar is higher in frequency and the pseudodiatomic potential has a greater well depth. This indicates that the interaction is stronger between 2,6-difluoropyrine and Ar than in the other complexes. The same trend is seen in the counterpoise corrected complexation energies from the ab initio calculations although the 
values are all on the order of $213 \mathrm{~cm}^{-1}$ and differ by only thousandeths of $\mathrm{cm}^{-1}$ rather than tens of $\mathrm{cm}^{-1}$ as for the experimentally derived well depths in Table 4 . The electrostatic potential energy surfaces and the results of the ${ }^{14} \mathrm{~N}$ hyperfine analysis for difluoropyrines in reference 12 demonstrated that fluorine substitution at both of the ortho carbon positions provides increased electron density at the nitrogen atom through a hyperconjugation effect as the fluorine atoms donate electron density into the $\pi$-system of the ring. Thus, it is not surprising to find among all the ab initio structures of the dimers, that the Ar atom in the complex with 2,6-difluoropyrine sits closest to both the aromatic ring plane and nitrogen atom $(3.51 \AA$ and $3.67 \AA)$ in comparison to the other species studied $(3.51 \AA$ and $3.69 \AA ; 3.53 \AA$ and $3.73 \AA ; 3.51 \AA$ and $3.69 \AA ; 3.53 \AA$ and $3.74 \AA$ for 2,3-, 2,4-, 2,5- and 3,5- difluoropyrine-Ar respectively).

Besides the stretching mode of the difluorinated pyridine-Ar dimers, it is informative to evaluate the two bending modes around the monomer's principal axes that lie in the ring plane. For the parent pyridine-Ar and furan-Ar complexes with one symmetry plane (aligned with the $a^{\prime} c^{\prime}$ plane of the monomers), Gutowsky and coworkers ${ }^{1}$ introduced a method to estimate the ring oscillation about the in-plane axes, which can also apply to our 2,6- and 3,5-difluoropyrine-Ar dimers. Both 2,6- and 3,5difluoropyrine-Ar dimers are nearly perfect asymmetric tops (the asymmetric parameter $\kappa$ -0.08 and 0.002 respectively) with the Ar atom residing in the symmetry plane ( $b^{\prime} c^{\prime}$ plane of the monomer, also the $a c$ plane of the dimer). In this case, the planar moment $P_{\mathrm{b}}$ of the dimer should equal the planar moment $P_{\mathrm{a}^{\prime}}$ of the monomer for a rigid system. For both 2,6- and 3,5-difluoropyrine-Ar complexes, however, the value of $P_{\mathrm{b}}$ is consistently 
smaller than the $P_{\mathrm{a}^{\prime}}$ of the corresponding free monomer, which is indicative of the intermolecular bending vibration of the rigid aromatic ring within the complex.

Before further deductions for these two dimers, we adopt a new axis frame (x, y, z) for both monomer and dimer (x: $a^{\prime}$ axis, $\mathrm{y}: b^{\prime}$ axis, and $\mathrm{z}: c^{\prime}$ axis of the monomer) for simplification. Rotation of the monomer about the $a^{\prime}$ (x) axis (also $b$ axis of the dimer) does not affect $P_{\mathrm{b}}$, but rotation by a small angle $\beta_{\mathrm{y}}$ about the $b^{\prime}(\mathrm{y})$ axis reduces $P_{\mathrm{b}}$ to:

$$
P_{b}=P_{a^{\prime}} \cos ^{2} \beta_{y}
$$

Rotation by a small angle $\beta_{\mathrm{x}}$ of the aromatic ring about the $a^{\prime}$ (x) axis should have a similar effect on $P_{\mathrm{c}}$ of the dimer. A small shift of both the $a$ and $c$ axes from the $c^{\prime}$ and $b^{\prime}$ axes, respectively, however makes the evaluation of the angle $\beta_{\mathrm{x}}$ difficult. Instead, one can evaluate the "average" angular displacement $\beta$ of the $c^{\prime}$ axis from its equilibrium position in the complex since the angle $\beta$ is related to $\beta_{\mathrm{x}}$ and $\beta_{\mathrm{y}}$ by the trigonometric relationship:

$$
\tan ^{2} \beta=\tan ^{2} \beta_{x}+\tan ^{2} \beta_{y}
$$

In order to deduce $\beta$, the inertial tensor of the dimer in the $(\mathrm{x}, \mathrm{y}, \mathrm{z})$ frame can be expressed:

$$
\begin{aligned}
& I_{x x}=I_{a^{\prime}}\left(1+\frac{1}{2} \sin ^{2} \beta\right)+\mu R^{2} \\
& I_{y y}=I_{b^{\prime}}\left(1+\frac{1}{2} \sin ^{2} \beta\right)+\mu R^{2} \cos ^{2} \gamma \\
& I_{z z}=I_{c^{\prime}}\left(1-\frac{1}{2} \sin ^{2} \beta\right)+\mu R^{2} \sin ^{2} \gamma \\
& I_{y z}=-\mu R^{2} \sin \gamma \cos \gamma
\end{aligned}
$$


where $\mu, R$ and $\gamma$ are defined as the reduced mass of the dimer when treated as two pseudodiatomic subunits; the distance from the $\mathrm{CM}$ of the monomer to the Ar atom; and the angle between the $\mathrm{c}^{\prime}$ axis of the monomer and the Ar-CM line, respectively. Because $\mathrm{x}\left(a^{\prime}\right)$ axis coincides with the principal $b$ axis of the dimer $\left(I_{\mathrm{xx}}=I_{\mathrm{b}}\right)$, diagonalization of the $2 \times 2$ block containing $I_{y z}$ will yield $I_{\mathrm{a}}$ and $I_{\mathrm{c}}$ of the dimer. As we require the angle $\beta$, the calculation, however, can be simplified by adding Eq. $4 \mathrm{~b}$ and Eq. $4 \mathrm{c}$ together:

$$
I_{y y}+I_{z z}=I_{b^{\prime}}+I_{c^{\prime}}+\frac{1}{2} \sin ^{2} \beta \times\left(I_{b^{\prime}}-I_{c^{\prime}}\right)+\mu R^{2}
$$

For matrix operations, the trace of the matrix should not change before and after the diagonalization such that $I_{\mathrm{yy}}+I_{\mathrm{zz}}=I_{\mathrm{a}}+I_{\mathrm{c}}$. With these relationships and the known inertial moments of the monomer and the dimer, one can simply use Eq. 4a and Eq. 4e to solve for $\beta$ and $R$. For both 2,6- and 3,5- difluoropyrine-Ar complexes, this approach provides exactly the same $R$ values as the RGDFIT method listed in Table 2 and the bending angles determined are listed in Table 5. The analogous angular parameters of pyridine-Ar and furan-Ar from Gutowsky and coworkers are also listed for comparison. ${ }^{1}$ From the furan-Ar dimer to the pyridine-Ar complexes, one observes a decrease in the angular displacement of the aromatic ring around the in-plane axes with increasing moments of inertia of the ring monomer. For both furan-Ar and pyridine-Ar, the bending angle $\beta_{\mathrm{x}}$ is almost the same as $\beta_{\mathrm{y}}$ (about $9^{\circ}$ for furan-Ar and $5^{\circ}$ for pyridine-Ar), which may be a consequence of the similar inertial moments of the monomer around the in-plane axes $\left(I_{\mathrm{a}^{\prime}}\right.$ $=53.5 \mathrm{amu} \cdot \AA$ and $I_{\mathrm{b}^{\prime}}=54.6 \mathrm{amu} \cdot \AA$ for furan; $I_{\mathrm{a}^{\prime}}=83.7 \mathrm{amu} \cdot \AA$ and $I_{\mathrm{b}^{\prime}}=87.1 \mathrm{amu} \cdot \AA$ for pyridine). For both 2,6- and 3,5- difluoropyrine-Ar complexes in this work, the bending angle $\beta_{\mathrm{x}}$ is almost twice that of $\beta_{\mathrm{y}}\left(4.5^{\circ}\right.$ and $2.6^{\circ}$ for 2,6 - difluoropyrine-Ar; $4.4^{\circ}$ and $2.4^{\circ}$ for 3,5- difluoropyrine-Ar) which is a reflection of the monomers' moments of 
inertia around the in-plane axes $\left(I_{\mathrm{a}^{\prime}}=134.8 \mathrm{amu} \cdot \AA\right.$ and $I_{\mathrm{b}^{\prime}}=265.2 \mathrm{amu} \cdot \AA$ for $2,6-$ difluoropyrine; $I_{\mathrm{a}^{\prime}}=131.2 \mathrm{amu} \cdot \AA \AA$ and $I_{\mathrm{b}^{\prime}}=283.2 \mathrm{amu} \cdot \AA$ for 3,5 - difluoropyrine). The bending angles of the 2,6-difluorpyridine-Ar and 3,5-difluoropyridine complexes are similar to each other suggesting that the trends observed in the stretching mode do not carry over to the bending coordinates. The bending angles for these difluorinated species are smaller than those of the furan-Ar and pyridine-Ar complexes indicating that the former are more rigid in the angular coordinates. This is not surprising as the substitution of fluorine atoms off-axis increases the moments of inertia for the monomers substantially. Unfortunately, it is difficult to apply the same method to other difluoropyrine-Ar complexes due to their reduced symmetry.

\section{Acknowledgements}

Funding for this research was provided by the Natural Sciences and Engineering Research Council (NSERC) of Canada through the Discovery Grant programme.

\section{Supporting Information Available}

The assigned transition frequencies for the five complexes are provided as supplementary material. This information is available free of charge via the Internet at http://pubs.acs/org. 
Table 1. Spectroscopic Constants of the difluoropyridine-Ar dimers.

\begin{tabular}{|c|c|c|c|c|c|}
\hline & 2,3-difluoropyridine-Ar & 2,4-difluoropyridine-Ar & 2,5-difluoropyridine-Ar & 2,6-difluoropyridine-Ar & 3,5-difluoropyridine-Ar \\
\hline \multicolumn{6}{|c|}{ Rotational Constants /MHz } \\
\hline $\mathrm{A}$ & $1415.50558(11)$ & $1266.103839(78)$ & $1214.51424(11)$ & $1269.979130(82)$ & $1238.55083(15)$ \\
\hline $\mathrm{B}$ & $948.258207(55)$ & $1000.803678(54)$ & $1095.774295(91)$ & $1019.355086(84)$ & $1001.67680(15)$ \\
\hline $\mathrm{C}$ & $856.762474(63)$ & $768.749795(69)$ & $713.718761(95)$ & $805.89569(29)$ & $763.57649(54)$ \\
\hline \multicolumn{6}{|c|}{ Centrifugal Distortion Quartic Constants /kHz } \\
\hline$\Delta_{\mathrm{J}}$ & $2.0000(10)$ & $1.8992(14)$ & $3.6424(29)$ & $1.3465(60)$ & $1.441(16)$ \\
\hline$\Delta_{\mathrm{JK}}$ & $4.0738(48)$ & $4.4421(55)$ & $-8.838(11)$ & $10.369(18)$ & $7.622(50)$ \\
\hline$\Delta_{\mathrm{K}}$ & $-3.8732(65)$ & $-3.9546(48)$ & $12.0923(95)$ & $-11.651(12)$ & $-8.792(34)$ \\
\hline$\delta_{\mathrm{j}}$ & $0.39852(11)$ & $0.56405(21)$ & $1.57595(56)$ & $0.2847(28)$ & $0.3157(80)$ \\
\hline$\delta_{\mathrm{k}}$ & $1.7064(22)$ & $3.3683(14)$ & $0.2427(18)$ & $4.2035(93)$ & $3.711(25)$ \\
\hline \multicolumn{6}{|c|}{ Sextic Distortion Constants $/ \mathrm{Hz}$} \\
\hline$\Phi_{\mathrm{J}}$ & $-0.0382(56)$ & $-0.033(11)$ & $-0.294(30)$ & & \\
\hline$\Phi_{\mathrm{JJK}}$ & $0.546(45)$ & $0.513(66)$ & $4.27(19)$ & $-0.297(48)$ & $-0.29(12)$ \\
\hline$\Phi_{\mathrm{JKK}}$ & $-1.58(12)$ & $-1.57(10)$ & $-11.54(35)$ & $0.93(13)$ & $0.75(37)$ \\
\hline$\Phi_{\mathrm{K}}$ & $1.252(84)$ & $1.278(75)$ & $7.87(25)$ & $-0.66(11)$ & $-0.41(31)$ \\
\hline \multicolumn{6}{|c|}{${ }^{14} \mathrm{~N}$ Nuclear Quadrupole Coupling Constants /MHz } \\
\hline $1.5 \chi_{\mathrm{aa}}$ & $3.9658(30)$ & $2.6465(23)$ & $3.0076(29)$ & $3.3347(14)$ & $4.5608(53)$ \\
\hline $0.25\left(\chi_{\mathrm{bb}}-\chi_{\mathrm{cc}}\right)$ & $1.52743(40)$ & $-0.67172(40)$ & $0.97813(61)$ & $1.47658(36)$ & $1.52144(70)$ \\
\hline$\chi_{\mathrm{ab}^{\mathrm{a}}}$ & -0.249 & 1.613 & 1.177 & & \\
\hline$\chi_{\mathrm{ac}^{\mathrm{a}}}$ & 0.774 & 0.941 & 1.691 & -0.348 & 1.893 \\
\hline$\chi_{\mathrm{bc}}{ }^{\mathrm{a}}$ & -0.615 & -2.413 & -2.190 & & \\
\hline $\begin{array}{c}\text { rms error } \\
/ \mathrm{kHz}\end{array}$ & 3.4 & 2.8 & 3.3 & 2.3 & 2.7 \\
\hline no. lines & 352 & 295 & 240 & 227 & 152 \\
\hline
\end{tabular}

aThe off-diagonal parameters are fixed to those determined by MP2 calculations $(6-311++G(2 d, 2 p))$. 
Table 2. Structural Parameters of Aromatic Ring-Ar Dimers Obtained by Different Methods.

\begin{tabular}{|c|c|c|c|c|}
\hline & $\mathrm{R}(\AA)$ & $\gamma\left({ }^{\circ}\right)$ & $\theta\left(^{\circ}\right)$ & $\phi\left(^{\circ}\right)$ \\
\hline Pyridine-Ar ${ }^{\mathrm{a}}$ & 3.545 & 3.5 & 86.5 & 90.0 \\
\hline Furan-Ara & 3.541 & 5.5 & 84.5 & 90.0 \\
\hline \multicolumn{5}{|l|}{ 2.6-difluoropvridine- $\mathrm{Ar}$} \\
\hline method $\mathrm{I}^{\mathrm{b}}$ & 3.510 & 2 & 92 & 90 \\
\hline method II ${ }^{c}$ & 3.486 & 4.9 & 94.9 & 90 \\
\hline method III ${ }^{\mathrm{d}}$ & 3.486 & 2.0 & 92.0 & 90 \\
\hline \multicolumn{5}{|l|}{ 3,5-difluoropyridine-Ar } \\
\hline method I & 3.570 & 9 & 81 & 90 \\
\hline method II & 3.545 & 9.9 & 80.1 & 90 \\
\hline method III & 3.545 & 8.7 & 81.3 & 90 \\
\hline \multicolumn{5}{|l|}{ 2,3-difluoropyridine-Ar } \\
\hline method I & 3.560 & 10 & 82 & 84 \\
\hline method II & 3.534 & 10.0 & 81.4 & 84.9 \\
\hline method III & 3.534 & 8.5 & 83.1 & 85.0 \\
\hline \multicolumn{5}{|l|}{ 2,4-difluoropyridine-Ar } \\
\hline method I & 3.560 & 8 & 83 & 87 \\
\hline method II & 3.535 & 9.6 & 81.0 & 86.6 \\
\hline method III & 3.535 & 6.5 & 83.6 & 88.7 \\
\hline \multicolumn{5}{|l|}{ 2,5-difluoropyridine-Ar } \\
\hline method I & 3.520 & 4 & 86 & 89 \\
\hline method II & 3.505 & 8.4 & 81.7 & 88.6 \\
\hline method III & 3.505 & 3.9 & 86.1 & 89.4 \\
\hline
\end{tabular}

\footnotetext{
${ }^{a}$ Ref. 1, by analysis of the moments of inertia (method II).

${ }^{\mathrm{b}}$ Method I: from MP2 $(6-311++\mathrm{g}(2 \mathrm{~d}, 2 \mathrm{p}))$ calculations.

${ }^{\mathrm{c}}$ Method II: from inertial analysis (RGDFIT) with ring structures $\left(r_{0}\right)$ from Ref. 12

${ }^{\mathrm{d}}$ Method III: from quadrupole hyperfine analysis with R from RGDFIT and ring structures ( $\mathrm{r}_{0}$ ) from Ref. 12.
} 
Table 3. ${ }^{14} \mathrm{~N}$ nuclear quadrupole coupling constants of the fluoropyridines and their Ar dimers in MHz.

\begin{tabular}{|c|c|c|c|c|c|c|}
\hline & $\chi_{\mathrm{aa}}$ & $\chi_{\mathrm{bb}}$ & $\chi_{\mathrm{cc}}$ & $\chi_{\mathrm{ab}}$ & $\chi_{\mathrm{ac}}$ & $\chi_{\mathrm{bc}}$ \\
\hline 2,3-difluoropyridine ${ }^{a}$ & 1.658 & -4.598 & 2.940 & & & \\
\hline \multicolumn{7}{|l|}{ 2,3-difluoropyridine-Ar } \\
\hline experiment $^{\mathrm{b}}$ & 2.644 & 1.733 & -4.377 & -0.249 & 0.774 & -0.615 \\
\hline method $\mathrm{I}^{\mathrm{c}}$ & 2.589 & 1.810 & -4.399 & -0.249 & 0.774 & -0.615 \\
\hline method II ${ }^{\mathrm{d}}$ & 2.651 & 1.707 & -4.358 & -0.275 & 1.087 & -0.736 \\
\hline 2,4-difluoropyridine ${ }^{a}$ & -2.992 & 0.440 & 2.552 & 2.550 & & \\
\hline \multicolumn{7}{|l|}{ 2,4-difluoropyridine-Ar } \\
\hline experiment ${ }^{\mathrm{b}}$ & 1.764 & -2.226 & 0.461 & 1.613 & 0.941 & -2.413 \\
\hline method I & 2.033 & -2.518 & 0.485 & 1.613 & 0.941 & -2.413 \\
\hline method $\mathrm{II}^{\mathrm{d}}$ & 1.650 & -2.122 & 0.472 & 1.990 & 1.200 & -2.282 \\
\hline 2,5-difluoropyridine ${ }^{a}$ & 0.156 & -3.007 & 2.851 & 3.000 & & \\
\hline \multicolumn{7}{|l|}{ 2,5-difluoropyridine-Ar } \\
\hline experiment ${ }^{\mathrm{b}}$ & 2.005 & 0.954 & -2.959 & 1.177 & 1.691 & -2.190 \\
\hline method I ${ }^{\mathrm{c}}$ & 1.973 & 1.007 & -2.980 & 1.177 & 1.691 & -2.190 \\
\hline method II ${ }^{\mathrm{d}}$ & 1.591 & 1.252 & -2.843 & 1.343 & 2.354 & -2.041 \\
\hline 2,6-difluoropyridine ${ }^{\mathrm{a}}$ & 1.842 & -4.101 & 2.259 & & & \\
\hline \multicolumn{7}{|l|}{ 2,6-difluoropyridine-Ar } \\
\hline experiment $\mathrm{t}^{\mathrm{b}}$ & 2.223 & 1.842 & -4.065 & & -0.348 & \\
\hline method $\mathrm{I}^{\mathrm{c}}$ & 2.237 & 1.903 & -4.140 & & -0.348 & \\
\hline method II ${ }^{\mathrm{d}}$ & 2.142 & 1.842 & -3.985 & & -0.852 & \\
\hline 3,5-difluoropyridine ${ }^{a}$ & 1.516 & -5.035 & 3.519 & & & \\
\hline \multicolumn{7}{|l|}{ 3,5-difluoropyridine-Ar } \\
\hline experiment $^{\mathrm{b}}$ & 3.041 & 1.523 & -4.563 & & 1.893 & \\
\hline method I ${ }^{\mathrm{c}}$ & 2.846 & 1.674 & -4.521 & & 1.893 & \\
\hline method II ${ }^{\mathrm{d}}$ & 2.947 & 1.516 & -4.463 & & 2.137 & \\
\hline
\end{tabular}

${ }^{a}$ Ref. 12 (experiment).

${ }^{\mathrm{b}}$ Off-diagonal terms were fixed to $a b$ initio values during spectral analysis.

${ }^{c}$ Method I: from MP2 $(6-311++\mathrm{g}(2 \mathrm{~d}, 2 \mathrm{p}))$ calculations.

${ }^{\mathrm{d}}$ Method II: from rotating the monomer quadrupole tensor into the dimer axis system based on the RGDFIT analysis. 
Table 4. Lennard-Jones potential parameters obtained by pseudodiatomic approximation. ${ }^{\mathrm{a}}$

\begin{tabular}{cccccc}
\hline \hline & $R_{0}(\AA)^{\mathrm{d}}$ & $B_{0}(\mathrm{MHz})^{\mathrm{e}}$ & $k_{\mathrm{s}}\left(\mathrm{N} \cdot \mathrm{m}^{-1}\right)$ & $v_{\mathrm{s}}\left(\mathrm{cm}^{-1}\right)$ & $\epsilon\left(\mathrm{cm}^{-1}\right)$ \\
Pyridine-Ar $^{\mathrm{b}}$ & 3.545 & 1515.089 & 2.70 & 41.5 & 232.0 \\
Furan-Ar $^{\mathrm{c}}$ & 3.541 & 1505.979 & 2.69 & 42.5 & 236.0 \\
2,6-difluoropyridine-Ar & 3.486 & 1402.391 & 3.01 & 41.5 & 250.7 \\
3,5-difluoropyridine-Ar & 3.545 & 1355.522 & 2.59 & 38.5 & 223.0 \\
2,3-difluoropyridine-Ar & 3.534 & 1364.637 & 1.91 & 33.1 & 162.5 \\
2,4-difluoropyridine-Ar & 3.535 & 1363.857 & 1.97 & 33.6 & 167.5 \\
2,5-difluoropyridine-Ar & 3.505 & 1387.148 & 1.22 & 26.4 & 101.1 \\
\hline \hline
\end{tabular}

${ }^{a}$ This method as applied to a pseudodiatomic complex is described in Refs 3, 10 and 24.

${ }^{\mathrm{b}}$ Ref. 1 .

${ }^{\mathrm{c}}$ Ref. 3 .

${ }^{\mathrm{d}}$ The pseudoatomic bond length from Table 2 .

e The pseudoatomic rotational constants based on $R_{0}$.

Table 5. Angular Displacement $\beta$ of the Aromatic Ring From Its Equilibrium Position During the Bending Motion. ${ }^{\text {a }}$

\begin{tabular}{cccc} 
& $\beta\left({ }^{\circ}\right)^{\mathrm{b}}$ & $\beta_{\mathrm{x}}\left({ }^{\circ}\right)^{\mathrm{c}}$ & $\beta_{\mathrm{y}}\left({ }^{\circ}\right)^{\mathrm{d}}$ \\
Furan-Ar $^{\mathrm{e}}$ & 12.2 & 8.7 & 8.7 \\
Pyridine-Ar & 7.2 & 5.2 & 5.0 \\
2,6-difluoropyridine-Ar & 5.2 & 4.5 & 2.6 \\
3,5-difluoropyridine-Ar & 5.0 & 4.4 & 2.4 \\
\hline
\end{tabular}

${ }^{\mathrm{a}}$ This method as applied to an aromatic ring-Ar complex with a symmetry plane is described in Ref. 1.

${ }^{\mathrm{b}}$ Average angular displacement of the $\mathrm{c}^{\prime}$ axis of the monomer from its equilibrium position.

${ }^{c}$ Angular displacement of the aromatic ring about the a' axis of the monomer.

d Angular displacement of the aromatic ring about the b' axis of the monomer.

${ }^{\mathrm{e}}$ Ref. 1. 


\section{Figure Captions}

Figure 1: Orientation of the difluoropyridine-Ar complexes in the principal inertial axis system of the dimers looking down on the ab-plane in each based on MP2 $(6-311++\mathrm{g}(2 \mathrm{~d}, 2 \mathrm{p}))$ calculations.

Figure 2: A portion of the cp-FTMW spectrum of 2,3-difluoropyridine in both Ar (top) and $\mathrm{Ne}$ (bottom) used to identify lines due to the dimer complex with Ar. Hyperfine splittings due to nitrogen are visible for some transitions and weak features due to the ${ }^{13} \mathrm{C}$ and ${ }^{15} \mathrm{~N}$ isotopologues are also observed. Unidentified lines are marked by asterisks.

Figure 3: Sample Balle-Flygare FTMW spectrum of the $43,2-32,2$ transition of the 2,3-difluoropyridine-Ar complex showing the hyperfine splitting due to the ${ }^{14} \mathrm{~N}$ quadrupole nucleus.

Figure 4: Definitions of the van der Waals coordinates of the dimer complexes in relation to the monomer $\mathrm{a}^{\prime}, \mathrm{b}^{\prime}, \mathrm{c}^{\prime}$-axis system. The $\mathrm{R}$ coordinate corresponds to the Ar-CM separation. 
Figure 1. Sun et al.
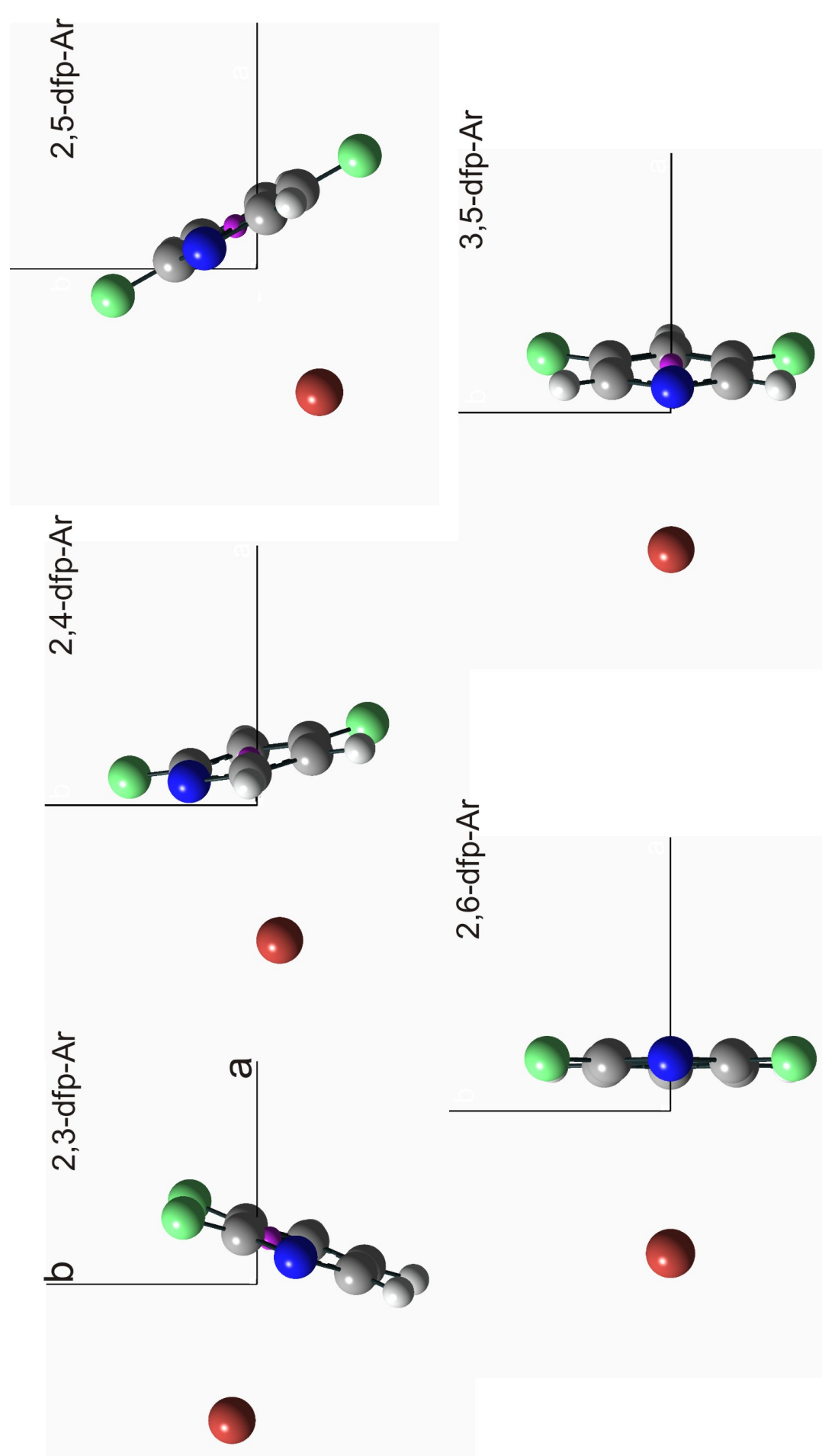
Figure 2. Sun et al.

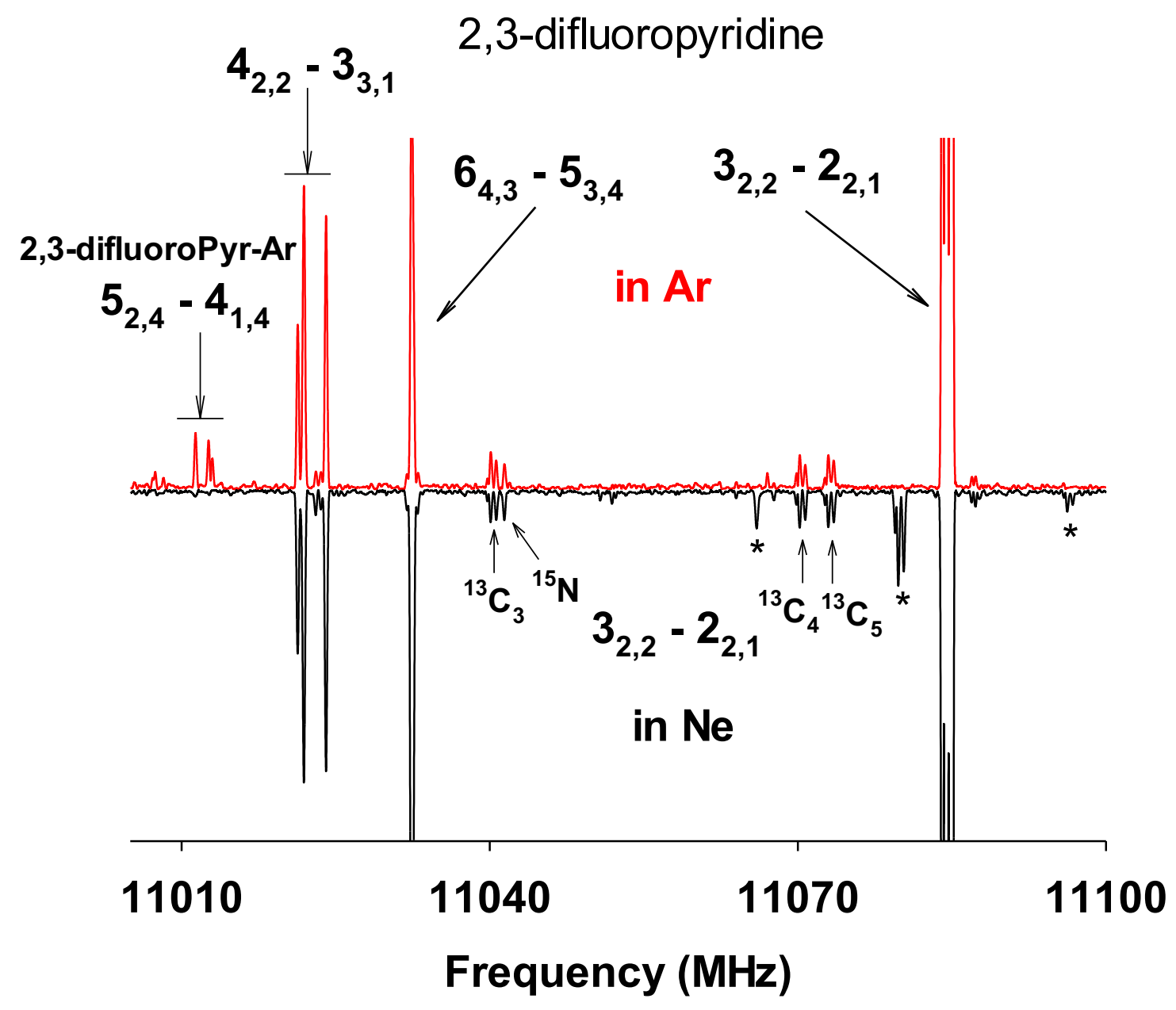


Figure 3. Sun et al.

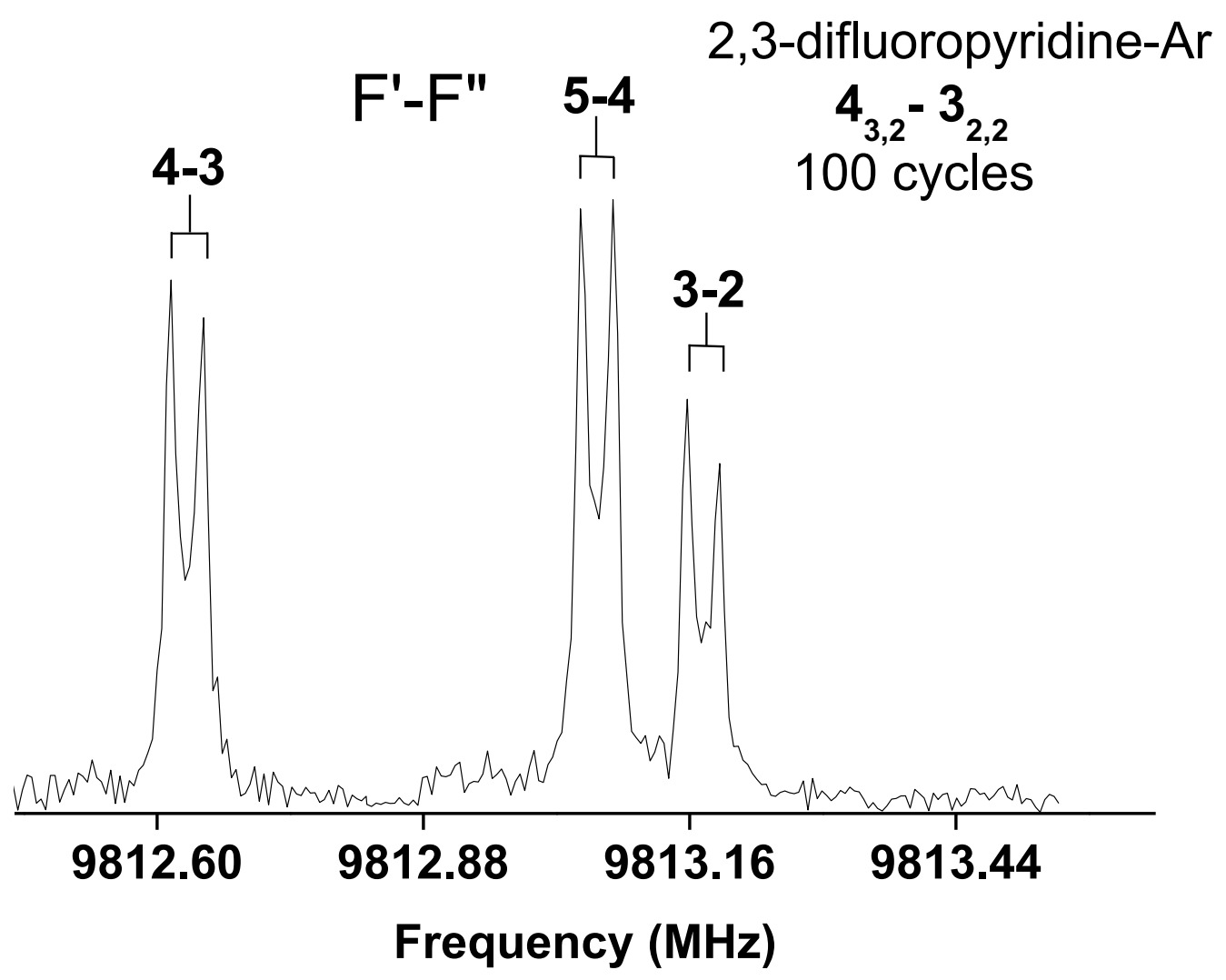


Figure 4. Sun et al.

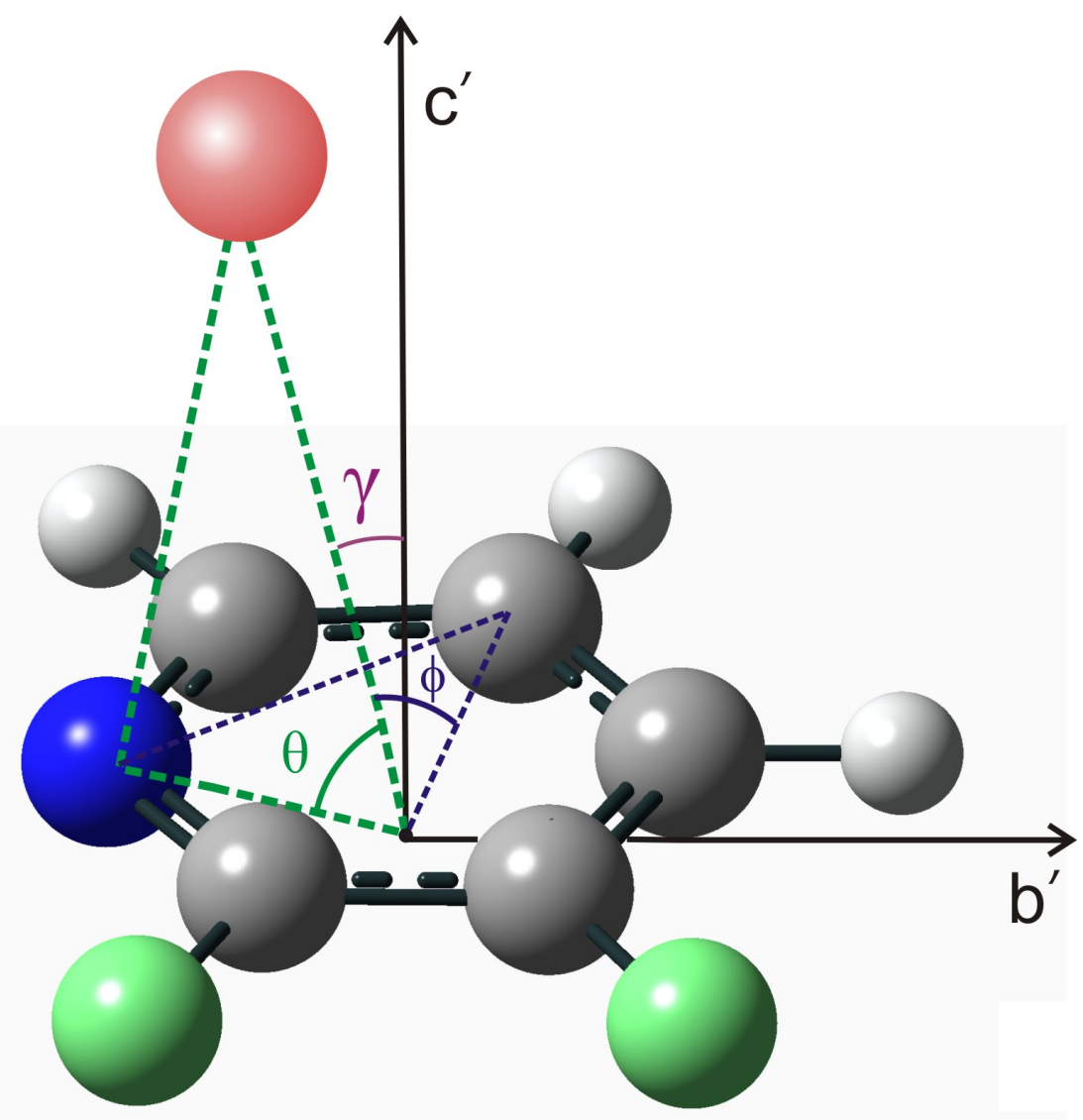


TOC figure. Sun et al.

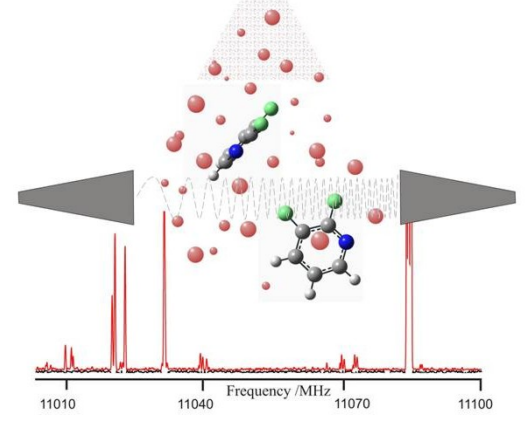




\section{References}

1 Klots, T. D.; Emilsson, T.; Ruoff, R. S.; Gutowsky, H. S. Microwave Spectra of Noble Gas-Pyridine Dimers:Argon-Pyridine and Kr-Pyridine. J. Phys. Chem. 1989, 93, 1255-1261.

2 Spycher, R. M.; Petitprez, D.; Bettens, F. L.; Bauder, A. Rotational Spectra of Pyridine-(Argon)n, n=1,2 Complexes and Their Vibrationaly Averaged Structures. J. Phys. Chem. 1994, 98, 11863-11869.

3 Kukolich, S. G. Microwave Structure Measurements on the Furan-Argon Complex. $J$. Am. Chem. Soc. 1983, 105, 2207-2210.

${ }^{4}$ Kraka, E.; Cremer, D. Intermolecular Forces in Argon van der Waals Complexes. Rotational Spectrum and Ab Initio Investigation of Ar-Oxazole. J. Phys. Chem. 1995, 99, 12466-12477.

5 Oh, J. J.; Park, I.; Wilson, R. J.; Peebles, S. A.; Kuczkowski, R. L.; Kraka, E.; Cremer, D. Structure of the Chlorobenzene-Argon Dimer: Microwave Spectrum and ab initio Analysis. J. Chem. Phys. 2000, 113, 9051-9059.

6 Neill, J. L.; Shipman, S. T.; Alvarez-Valtierra, L.; Lesarri, A.; Kisiel, Z.; Pate, B. H. Rotational Spectroscopy of Iodobenzene and Iodobenzene-Neon with a Direct Digital 2-8 GHz Chirped-Pulse Fourier Transform Microwave Spectrometer. J. Mol. Spectrosc. 2011, 269, 21-29.

7 Velino, B.; Caminati, W. Fourier Transform Microwave Spectrum of Pyridine-Neon. $J$. Mol. Spectrosc. 2008, 251, 176-179.

8 Evangelisti, L.; Favero, L. B.; Giuliano, B. M.; Tang, S.; Melandri, S.; Caminati, W. Microwave Spectrum of [1,1]-Pyridine-Ne2. J. Phys. Chem. A 2009, 113, 14227-14230.

9 Tanjaroon, C; Jäger, W. High-resolution Microwave Spectrum of the Weakly Bound Helium-Pyridine Complex. J. Chem. Phys. 2007, 127, 034302.

10 Sun, M.; Kamaee, M.; van Wijngaarden, J. Rotational Spectra and Structures of the van der Waals Dimers of Argon with 2-Fluoropyridine and 3-Fluoropyridine. J. Phys. Chem. A 2013, 117, 13429-13434.

11 van Dijk, C. W.; Sun, M.; van Wijngaarden, J. Microwave Rotational Spectra and Structures of 2-Fluoropyrine and 3-Fluoropyridine. J. Phys. Chem. A 2012, 116, 4082-4088. 
12 van Dijk, C. W.; Sun, M.; van Wijngaarden, J. Investigation of Structural Trends in Difluoropyridine Rings Using Chirped-Pulse Fourier Transform Microwave Spectroscopy and Ab Initio Calculations. J. Mol. Spectrosc. 2012, 280, 34-41.

13 Evangelisti, L.; Sedo, G.; van Wijngaarden, J. Rotational Spectrum of 1,1,1-Trifluoro-2-butanone Using Chirped-Pulse Fourier Transform Microwave Spectroscopy. J. Phys. Chem. A. 2011, 115, 685-690.

${ }^{14}$ Sedo, G.; van Wijngaarden, J. Fourier Transform Microwave Spectra of a "New" Isomer of OCS-CO 2 . J. Chem. Phys. 2009, 131, 044303.

15 Frisch, M. J.; Trucks G. W.; Schlegel, H. B.; Scuseria, G. E.; Robb, M. A.; Cheeseman, J. R.; Montgomery Jr., J. A.; Vreven, T.; Kudin, K. N.; Burant, J. C. et al., Gaussian 09, Revision A.02, Gaussian, Inc.: Wallingford CT, 2009.

${ }^{16}$ Pickett, H. M. The Fitting and Prediction of Vibration-Rotation Spectra with Spin Interactions. J. Mol. Spectrosc. 1991, 148, 371-377.

17 Kisiel, Z. PROSPE - Programs for Rotational SPEctroscopy, http://info.ifpan.edu.pl/ kisiel/prospe.htm.

${ }^{18}$ Suenram, R. D.; Fraser, G. T.; Lovas, F. J.; Gillies, C. W.; Zozom, J. Microwave Spectrum, Structure, and Electric Dipole Moment of the Ar-Formamide van der Waals Complex. J. Chem. Phys. 1988, 89, 6141-6146.

19 Kisiel, Z.; Fowler, P. W.; Legon, A. C. Rotational Spectra and Structures of the van der Waals Dimers of Ar with a Series of Fluorocarbons: Ar...CH $\mathrm{CH}_{2} \mathrm{CHF}$, $\mathrm{Ar} . . . \mathrm{CH}_{2} \mathrm{CF}_{2}$, and Ar...CHFCF 2. J. Chem. Phys. 1991, 95, 2283-2291.

${ }^{20}$ Kraitchman, J. Determination of Molecular Structure from Microwave Spectroscopic Data. Am. J. Phys. 1953, 21, 17-24.

${ }^{21}$ Lin, W.; Novick, S. E. The Microwave Spectrum and Structure of the Argon Trifluoroacetonitrile Complex. J. Mol. Spectrosc. 2007, 243, 32-36.

${ }^{22}$ Blanco, S.; Lister, D. G.; Legon, A. C.; Rego, C. A. Microwave Spectrum and Geometry of the Methyl Isocyanide-Argon Complex. J. Chem. Soc., Faraday Trans. 1997, 93, 1287-1290.

${ }^{23}$ Balle, T. J.; Campbell, E. J.; Keenan, K. R.; Flygare, W. H. A New Method for Observing the Rotational Spectra of Weak Molecular Complexes: KrHCl. J. Chem. Phys. 
1980, 72, 922-932.

${ }^{24}$ Read, W. G.; Campbell, E. J.; Henderson, G. The Rotational Spectrum and Molecular Structure of the Benzene-Hydrogen Chloride Complex. J. Chem. Phys. 1983, 78, 3501-3508. 